\title{
Standard Model Higgs Searches at the Tevatron
}

\author{
Nikos Varelas ${ }^{* \dagger}$ \\ University of Illinois at Chicago, Department of Physics, Chicago, IL 60607, USA \\ E-mail: varelaseuic.edu
}

\begin{abstract}
We review the status of searches for the Standard Model (SM) Higgs boson from the CDF and DØ experiments using a Run II data sample of $\approx 400 \mathrm{pb}^{-1} p \bar{p}$ collisions at center of mass energy of $1.96 \mathrm{TeV}$ at the Fermilab Tevatron. No evidence for signal is found and updated limits on the SM Higgs production cross section times branching ratio are presented. Studies of $W$ and $Z$ bosons produced in association with jets (background processes to SM Higgs production) are also discussed.
\end{abstract}

International Europhysics Conference on High Energy Physics

July 21st - 27th 2005

Lisboa, Portugal

${ }^{*}$ Speaker.

$\dagger$ Representing the $\mathrm{D} \varnothing$ and CDF Collaborations 


\section{Introduction}

One of the primary goals of present and future colliders is to discover the mechanism responsible for the spontaneous symmetry breaking of the $\mathrm{SU}(2)_{L} \times \mathrm{U}(1)_{Y}$ electroweak interaction. The simplest model for this mechanism is the standard Higgs model, based on a doublet of fundamental scalar fields. The current best limit on the mass of a Standard Model (SM) Higgs boson, $H$, is $M_{H}>114.4 \mathrm{GeV}$, at the $95 \%$ C. L. limit from the LEP experiments [1]. The recent global fit to electroweak precision data yields an expectation of the Higgs boson mass of $91_{-32}^{+45} \mathrm{GeV}$ with an upper limit of $219 \mathrm{GeV}$ at $95 \%$ C. L. [2]. These results suggest that the Higgs boson mass may not be very large and add urgency to Higgs boson searches at the Tevatron.

\section{Studies of Benchmark Processes}

Both CDF and DØ have performed measurements of final states related to background processes of the SM Higgs searches. Both experiments rely on their excellent electron and muon identification to trigger on $W$ and $Z$ final states. CDF used a sample of $127 \mathrm{pb}^{-1}$ to measure the $W+n \geq 0$ to 4 jet cross sections for jets reconstructed with a cone algorithm of $R_{\text {cone }}=0.4$, with transverse energy $E_{T}>15 \mathrm{GeV}$ and detector pseudorapidity $\left|\eta_{\text {det }}\right|<2.4$. The main sources of background, from QCD processes and from top production at the high multiplicity bins, vary from $4 \%$ to $40 \%$ from low to high jet multiplicity, respectively. D $\varnothing$ used a data sample of $343 \mathrm{pb}^{-1}$ to measure the ratios of the $Z / \gamma^{*}\left(\rightarrow e^{+} e^{-}\right)+\geq n$ jet cross sections to the total inclusive $Z / \gamma^{*} \rightarrow e^{+} e^{-}$ cross section for multiplicities $n \geq 0$ up to 5 jet events. DØ used jets with $R_{\text {cone }}=0.5$, with transverse momentum $p_{T}>20 \mathrm{GeV}$ and $|\eta|<2.5$. The CDF and D $\varnothing$ results are shown in Fig. 1 and compared to theoretical predictions.
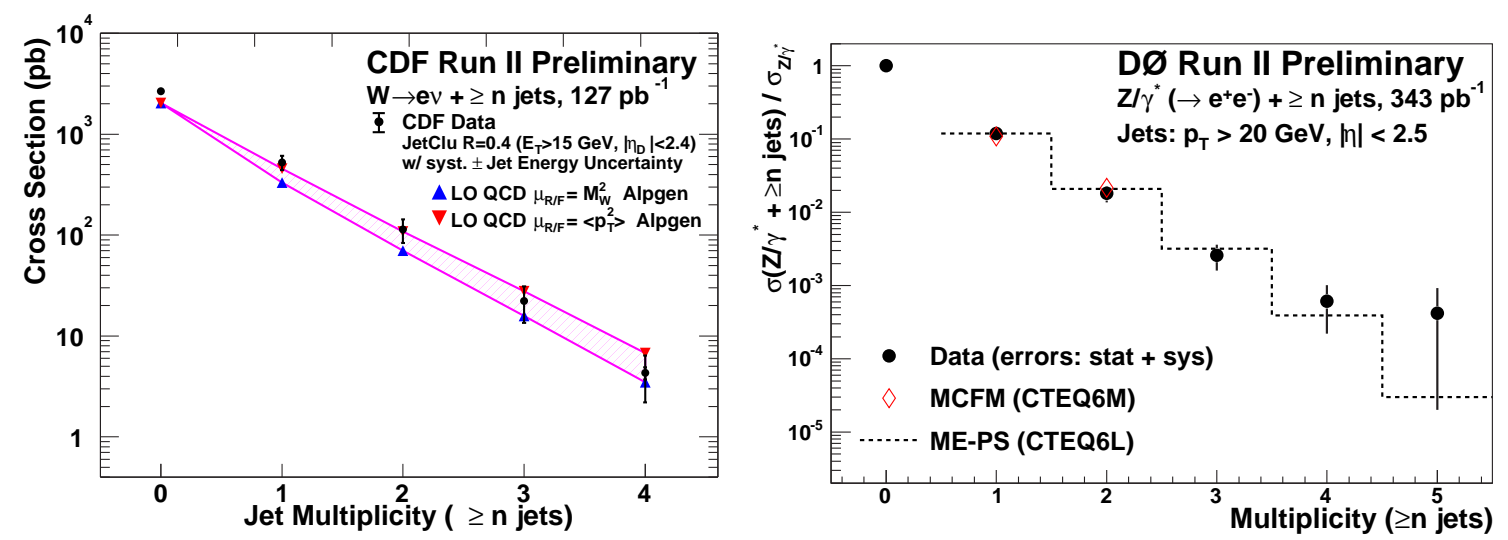

Figure 1: (left plot) $W+\geq n$ jet cross sections compared to ALPGEN [3] $W+n$ leading-order parton generator, with the evolution of partons into hadrons carried out by HERWIG followed by a full GEANT detector simulation. (right plot) Ratios of the $Z / \gamma^{*}\left(\rightarrow e^{+} e^{-}\right)+\geq n$ jet cross sections to the total inclusive $Z / \gamma^{*} \rightarrow e^{+} e^{-}$cross section versus $n$. The line represents the predictions [4] of leading-order Matrix Element (ME) calculations using PYTHIA for parton showering (PS) and hadronization, normalized to the measured $Z / \gamma^{*}+\geq 1$ jet cross section ratio. The open diamonds represent the next-to-leading-order predictions from MCFM [5]. 
CDF has observed a signal of about $3000 Z \rightarrow b \bar{b}$ decays using a sample of $333 \mathrm{pb}^{-1}$ of nearly back-to-back b-tagged jet events with $E_{T}>10 \mathrm{GeV}$. Such a sample could provide constraints to the b-jet energy calibration and be used in studies to improve the dijet mass resolution, increasing the chance of a Higgs discovery in the $b \bar{b}$ decay channel.

$\mathrm{D} \emptyset$ using a sample of $180 \mathrm{pb}^{-1}$ has performed a measurement of the ratio of inclusive cross sections for $p \bar{p} \rightarrow Z+b$ jet to $p \bar{p} \rightarrow Z+$ jet production. The $Z+b$ production is a source of background for the $Z H$ search in addition to being a benchmark process for the non-SM associated production of Higgs with b-quarks. Combining the $e e$ and $\mu \mu$ decay modes, and using jets with $p_{T}>20 \mathrm{GeV}$ and $|\eta|<2.5$, DØ measured a ratio $\sigma(p \bar{p} \rightarrow Z+b$ jet $) / \sigma(p \bar{p} \rightarrow Z+$ jet $)=$ $0.021 \pm 0.004$ (stat) ${ }_{-0.003}^{+0.002}$ (syst) which is in good agreement with the next-to-leading order prediction of $0.018 \pm 0.004[6]$.

\section{Low Mass Standard Model Higgs Searches}

The dominant production mode for the SM Higgs boson at the Tevatron is gluon fusion $g g \rightarrow$ $H$. However, for a light Higgs $\left(m_{H}<140 \mathrm{GeV}\right)$ the dominant decay mode is $H \rightarrow b \bar{b}$, making this mode hopeless due to the large backgrounds of $b \bar{b}$ production from other sources. The most promising modes for a light Higgs discovery at the Tevatron are those where the Higgs is produced in association with a $W$ or $Z$ boson, with $(W / Z) \rightarrow$ leptons and $H \rightarrow b \bar{b}$.

Both D $\varnothing$ and CDF have studied the $W b \bar{b}$ final state, in which the $W H$ production with $H \rightarrow b \bar{b}$ could be observed. DØ has published an earlier search for $W b \bar{b}$ and $W H$ production based on a $174 \mathrm{pb}^{-1}$ data sample from Run II [7]. Recently using a larger data sample of $382 \mathrm{pb}^{-1}$, DØ finds $13 \mathrm{~W} \rightarrow e v$ candidate events with $2 b$-tagged jets of $p_{T}>20 \mathrm{GeV}$ and $|\eta|<2.5$. With the number of expected events being 10.2, a 95\% C.L. upper cross section limit of $4.6 \mathrm{pb}$ is set on $W b \bar{b}$ production for $p_{T}^{b}>20 \mathrm{GeV},\left|\eta^{b}\right|<2.5$, and an $\eta-\phi$ separation between $b$-jets of $\Delta R_{b \bar{b}}>0.75$. By restricting the selection to a $\pm 25 \mathrm{GeV}$ window around the Higgs mass, DØ establishes a $95 \%$ C.L. limit on the $\sigma_{W H} \times B r(H \rightarrow b \bar{b})$ for Higgs masses in the range of $105-135 \mathrm{GeV}$ (see Fig. 2). CDF performed a similar search based on a $319 \mathrm{pb}^{-1}$ sample using $W \rightarrow l v(l=e, \mu)$ candidates and two jets, with $p_{T}>15 \mathrm{GeV}$ and $|\eta|<2$, one of which was a b-tagged jet. The CDF 95\% C.L. limits for $M_{H}=115$ to $135 \mathrm{GeV}$ are also shown in Fig. 2.

$\mathrm{D} \emptyset$ and CDF have searched for a SM Higgs produced in association with a $Z$ boson in the channel $Z(\rightarrow v \bar{v}) H(\rightarrow b \bar{b})$, which has comparable sensitivity to the $W(l v) H(b \bar{b})$ channel due to the large branching ratio of $Z \rightarrow v \bar{v}$. The $\mathrm{D} \emptyset$ analysis is based on a $261 \mathrm{pb}^{-1}$ data sample, collected using a trigger that selected events with acoplanar jets and missing transverse energy $\mathbb{Z}_{T}$. Events were selected with two non back-to-back $b$-tagged jets with $p_{T}>20 \mathrm{GeV}$ and $|\eta|<2.5$ with $E_{T}>25 \mathrm{GeV}$. To reduce contamination from $t \bar{t}$ events, the scalar sum of the $p_{T}$ of the jets, $H_{T}$, was required to be less than $200 \mathrm{GeV}$. To eliminate $W$ and $Z$ leptonic decays, events with isolated tracks with $p_{T}>8 \mathrm{GeV}$ were vetoed. Additional rejection of multijet background was obtained by various kinematic variables (using calorimeter cells, tracks, or jets) which are highly correlated in the case of the $v \bar{v} b \bar{b}$ final state. By restricting the selection to a $\pm 25 \mathrm{GeV}$ window around the Higgs mass, DØ establishes a 95\% C.L. limit on the $\sigma_{Z H} \times B r(H \rightarrow b \bar{b})$ production cross section for Higgs masses in the range of 105-135 GeV (see Fig. 2). CDF also performed a search on the same channel using a $289 \mathrm{pb}^{-1}$ data sample. A blind analysis technique was used with a final 
selection of two jets with $E_{T}>40 \mathrm{GeV}$ and $E_{T}>20 \mathrm{GeV}$ for the leading and 2nd leading- $E_{T}$ jet respectively, with at least one $b$-tagged jet, and $E_{T}>70 \mathrm{GeV}$. The cross section time branching ratio limits from this analysis are shown in Fig. 2.

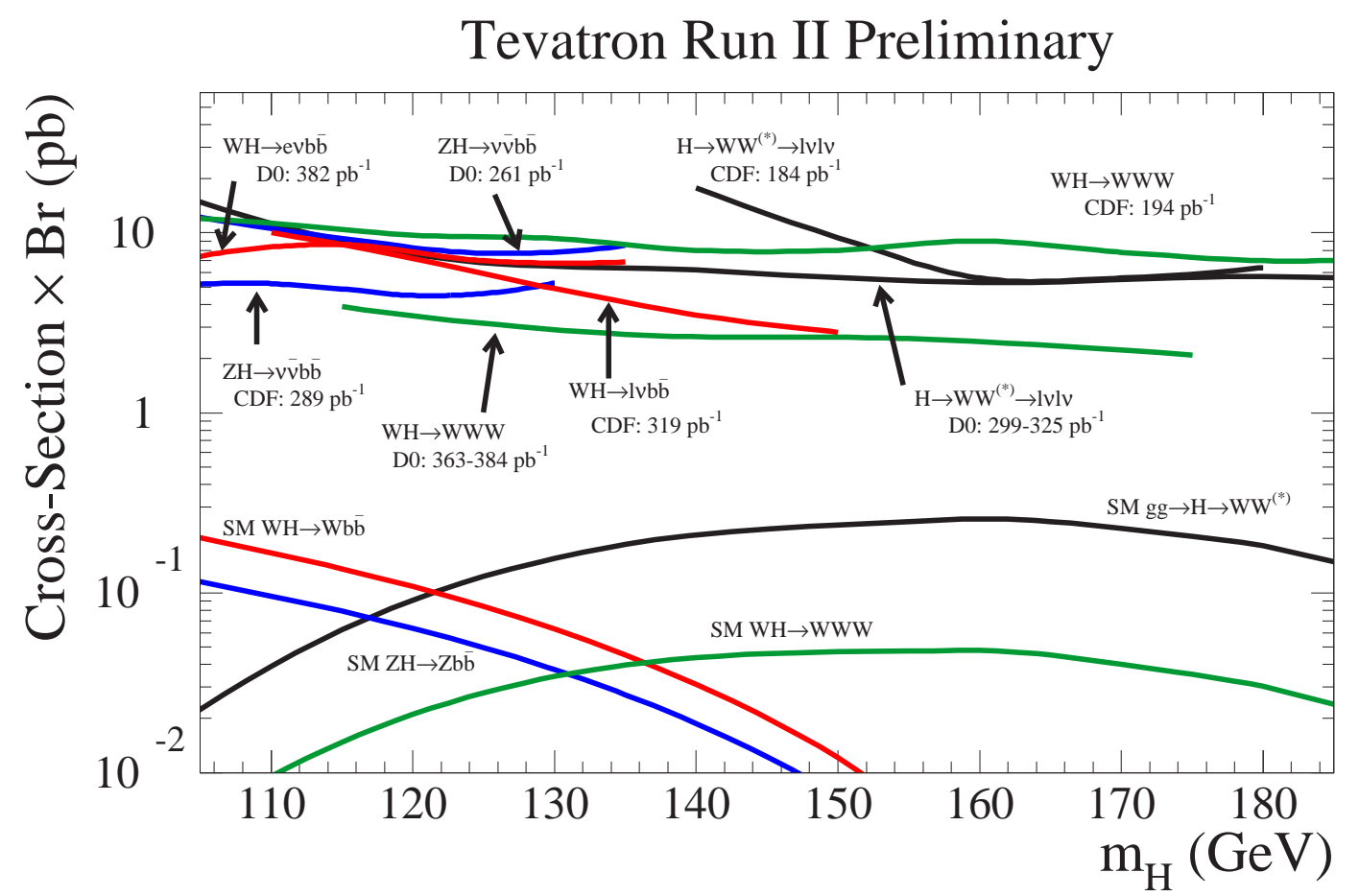

Figure 2: Summary of 95\% C.L. limits of the SM Higgs production cross section times branching ratio obtained by DØ and CDF from searches using Run II data as a function of the Higgs mass. The expected SM cross sections for the searched processes are also shown.

\section{High Mass Standard Model Higgs Searches}

For $M_{H}>140 \mathrm{GeV}$ the process $g g \rightarrow H \rightarrow W W^{*}$ becomes dominant. Since the dominant background process in this channel is $W W$ production, both experiments measured the $W W$ production cross section $[8,9]$. DØ and CDF combined both electron and muon decay channels of the $W$ bosons to search for a high mass Higgs. The basic event selection includes two high- $p_{T}$ oppositely charged leptons ( $e e, \mu \mu, e \mu$ channels), significant $E_{T}$, veto on energetic jets, and a dilepton invariant mass incompatible with a $Z$ boson and restricted to low values as expected from signal simulations. The azimuthal angle $\Delta \Phi_{l l}$ of the two charged leptons was used to reduce the non-resonant $W W$ background, since the zero spin of the Higgs boson and the helicity conservation of the $W$ decay products produce leptons in the same direction in the transverse plane (i.e small values of $\left.\Delta \Phi_{l l}\right)$. No signal is found and both CDF and DØ using a data sample of $184 \mathrm{pb}^{-1}$ and 
$\approx 320 \mathrm{pb}^{-1}$ respectively, set a 95\% C.L. limit for the $\sigma_{H} \times B r\left(H \rightarrow W W^{*}\right)$ as a function of Higgs mass as shown in Fig. 2.

Both CDF and DØ have also searched for the associated $W H\left(\rightarrow W W^{*}\right)$ production with three $W$ bosons at the final state. The event selection includes two same sign charged leptons (ee, $\mu \mu$, $e \mu$ channels) with significant $\not_{T}$. Both experiments use further cuts to reduce backgrounds and enhance the sensitivity to the Higgs signal, resulting in the 95\% C.L. limit for the production cross section times branching ratio as a function of Higgs mass shown in Fig. 2.

\section{Outlook}

Over the next few years the Tevatron experiments CDF and $\mathrm{D} \varnothing$ are in a unique position to search for the dynamics responsible for the electroweak symmetry breaking. The searches presented here from an initial data sample of approximately $400 \mathrm{pb}^{-1}$ up to Summer 2004, show no deviation from the Standard Model. With $8 \mathrm{fb}^{-1}$ of integrated luminosity expected by 2009, and with improved analyses and combined results among the various channels and between the two experiments, a light SM Higgs discovery is within reach, or its existence can be excluded over an interesting mass range.

\section{Acknowledgements}

I would like to thank my colleagues at $\mathrm{D} \emptyset$ and $\mathrm{CDF}$ for discussions of the results presented here.

\section{References}

[1] A. Heister et al. (The LEP Higgs Working Group), Phys. Lett. B 565, 62 (2003).

[2] http://lepewwg.web.cern.ch/LEPEWWG/

[3] M.L. Mangano, M. Moretti, F. Piccinini, R. Pittau, and A. Polosa, J. High Energy Phys. 07, 001 (2003).

[4] S. Mrenna and P. Richardson, JHEP 0405, 040 (2004) [arXiv:hep-ph/0312274].

[5] J. Campbell and R.K. Ellis, Phys. Rev. D 65, 113007 (2002).

[6] V. M. Abazov et al. (DØ Collaboration), Phys. Rev. Lett. 94, 161801 (2005).

[7] V. M. Abazov et al. (DØ Collaboration), Phys. Rev. Lett. 94091802 (2005).

[8] V. M. Abazov et al. (DØ Collaboration), Phys. Rev. Lett. 94151801 (2005).

[9] D. Acosta et al. (CDF Collaboration), Phys. Rev. Lett. 94211801 (2005). 